\title{
pH-Dependent Depletion Induced Phase Behavior of Silica Nanoparticles
}

\author{
Indresh Yadav ${ }^{1 *}$, V. K. Aswal ${ }^{1}$ and J. Kohlbrecher ${ }^{2}$ \\ ${ }^{1}$ Solid State Physics Division, Bhabha Atomic Research Centre, Mumbai 400 085, India \\ ${ }^{2}$ Laboratory for Neutron Scattering, Paul Scherrer Institut, CH-5232 PSI Villigen, Switzerland \\ ikyadav@barc.gov.in
}

\begin{abstract}
The addition of non-adsorbing macromolecules to a colloidal suspension is known to induce the depletion attraction between the particles whose strength and range depends on concentration and size of the macromolecules, respectively and also influenced by charge on the macromolecules. In this work we have studied the phase behavior of colloidal silica nanoparticles in presence of bovine serum albumin (BSA) protein at different $\mathrm{pH}$. Both the nanoparticles and BSA carry similar charges (negative), within the measured $\mathrm{pH}$ range, concomitantly the electrostatic repulsion prevents the adsorption of BSA on the nanoparticles. The nonadsorption of BSA induces the depletion attraction which leads the aggregation of the nanoparticles above a critical protein concentration (CPC). The CPC decreases with $\mathrm{pH}$ approaching towards the isoelectric points of BSA. This decrease in CPC is attributed to decrease in BSA-BSA repulsion which enhances the depletion attraction between the nanoparticles. The morphology of the aggregates, irrespective of the $\mathrm{pH}$, is found to be mass fractal.
\end{abstract}

Keywords: Nanoparticle-protein interaction, Depletion interaction, Phase behavior, DLS, SANS

PACS: 82.70.Dd, 64.70.-p, 87.15.nr, 61.05.fg, 61.46.Df,

\section{INTRODUCTION}

To study the interaction of the nanoparticles with proteins has attracted considerable attention due to its myriad applications in biology and material science. The interaction of the nanoparticles with proteins can control the protein-protein interaction, protein delivery, enzymatic activity, and be applied to diagnostics and sensors. In contact with biological fluids nanoparticles, due to the high surface-to-volume ratio, become coated with proteins and form a protein corona [1]. The adsorption of protein on the nanoparticles can impair the native structure of proteins and hence its biological functioning. The important aspects of nanoparticle-protein system are determined by the van der Waals, hydrogen bonding, steric repulsion, electrostatic and depletion interactions. In case of hydrophilic surfaces, if both the nanoparticles and protein are similarly charged, strong electrostatic repulsion prevents them from forming a conjugate and the protein structure almost remains intact [2]. The addition of non-adsorbing protein to a colloidal suspension of nanoparticles can induce the depletion attraction between the nanoparticles whose strength and range is proportional to concentration and size of protein molecules, respectively. The depletion attraction, at sufficient protein concentration, can leads to aggregation of the nanoparticles and thereby phase transformation. This phase behavior is mainly governed by the electrostatic interaction between different components (nanoparticle-nanoparticle, nanoparticle-protein and protein-protein) and can be tuned by $\mathrm{pH}$ [3]. Herein, we have studied the phase behavior of silica nanoparticles in presence of bovine serum albumin (BSA) protein with $\mathrm{pH}$ approaching towards the isoelectric point of BSA. The scattering techniques-dynamic light scattering (DLS) and smallangle neutron scattering (SANS) have been used to monitor the evolution of structure (kinetics) and the morphology of the aggregates in in-situ.

\section{EXPERIMENT}

The electrostatically stabilized colloidal suspensions of spherical silica nanoparticles (Ludox HS40) and lyophilized powder of BSA protein were purchased from Sigma-Aldrich. Samples were prepared in $20 \mathrm{mM}$ phosphate buffer at $\mathrm{pH} 7$ in $\mathrm{D}_{2} \mathrm{O}$ by keeping fixed concentration of silica nanoparticles (1 wt $\%)$ and varying the concentration (0-5 wt \%) of protein where $\mathrm{pH}$ was raised or lowered by adding $1 \mathrm{M}$ $\mathrm{NaOH}$ or $1 \mathrm{M} \mathrm{HCl}$, respectively. The DLS 
measurements were performed on nanoparticle size analyzer SZ-100 (HORIBA, Japan). Small-angle neutron scattering experiments were performed at the SANS-I facility at the Swiss spallation neutron source, SINQ, Paul Scherrer Institut, Switzerland [28]. All the measurements were carried out at $30^{\circ} \mathrm{C}$.

\section{DATA ANALYSIS}

The DLS is one of the most commonly used technique for measuring in situ the sizes and size distributions of the nanoparticles in colloidal suspensions. In this technique, the intensity autocorrelation function $\left[\mathrm{g}^{2}(\tau)\right]$ of scattered light as a function of delay time $(\tau)$ is measured. For ergodic systems, the autocorrelation function is related to the effective hydrodynamic size $\left(2 \mathrm{R}_{\mathrm{H}}\right)$ of the particles and can be given by [2]

$$
g^{2}(\tau)=\beta \exp \left(-2 \frac{k_{B} T}{6 \pi \eta R_{H}} Q^{2} \tau\right)+1
$$

where $\beta$ is an experimental constant. $k_{B}$ is the Boltzmann's constant, $\eta$ is the solvent viscosity, and $T$ is the absolute temperature.

In SANS, the coherent differential scattering cross section per unit volume $(d \Sigma / d \Omega)$ is measured as a function of momentum transfer $[Q=4 \pi \sin (\theta / 2) / \lambda$, where $\theta$ is the scattering angle and $\lambda$ is the wavelength]. For a collection of mono-disperse interacting particles it can be expressed; as [2]

$$
\frac{d \Sigma}{d \Omega}(Q)=\varphi V\left(\rho_{p}-\rho_{s}\right)^{2} P(Q) S(Q)+B
$$

where $V$ is volume of individual particle and $\varphi$ is their volume fraction. $\rho_{p}$ and $\rho_{s}$ are scattering length densities of particles and solvent, respectively. $P(Q)$ is the orientational average of the square of form factor, $F(Q)$, and gives information about shape and size of the particle. $S(Q)$ is interparticle structure factor which describes the correlation between the particles. $B$ is a constant term representing incoherent background.

\section{RESULTS AND DISCUSSION}

The pH-dependent phase behavior of the nanoparticles in presence of BSA protein is shown in Figure 1. The phase behavior is examined by measuring the transmission of light $(532 \mathrm{~nm})$ through silica nanoparticles ( $1 \mathrm{wt} \%$ ) as a function of protein concentration. Irrespective of the $\mathrm{pH}$, the phase behaviors show similar features where the transmission of light drops drastically beyond a particular protein concentration, referred as CPC. The $\mathrm{CPC}$ decreases with $\mathrm{pH}$ approaching towards the isoelectric point of BSA. Above CPC the well dispersed colloidal nanoparticles acquired protein induced aggregation. The formation of nanoparticle aggregates enhances the scattering of light and hence reduces the transmission [2]. The zeta potential measurement [inset in Figure 1] shows that the nanoparticles and BSA have the similar charge (negative) over the $\mathrm{pH}$ range from $\mathrm{pH} 9-\mathrm{pH} 5$ where isoelectric point of BSA is about $\mathrm{pH} 4.7$. Consequently, the dominance of electrostatic repulsion over site specific attraction seems to prevent the adsorption of BSA on the nanoparticles and leads to the depletion induced aggregation of the nanoparticles above CPC. It is also seen that the charge on the nanoparticles is same at $\mathrm{pH} 9$ and $\mathrm{pH} 7$ but reduces in significant amount at $\mathrm{pH} 5$, whereas for BSA it decreases monotonically with decrease in $\mathrm{pH}$. Thus with decrease in $\mathrm{pH}$ the electrostatic repulsion between different components (nanoparticle-nanoparticle, nanoparticle-protein and protein-protein) decreases. It is observed that the nanoparticle-protein repulsion is still enough to prevent the adsorption of BSA on the nanoparticles. The reduction in protein-protein repulsion enhances the entropy driven depletion interaction and is responsible for the decrease in CPC value. The quantitative study of the evolution of protein induced microstructures that decide the phase of nanoparticle-protein system has been studied by DLS and SANS.

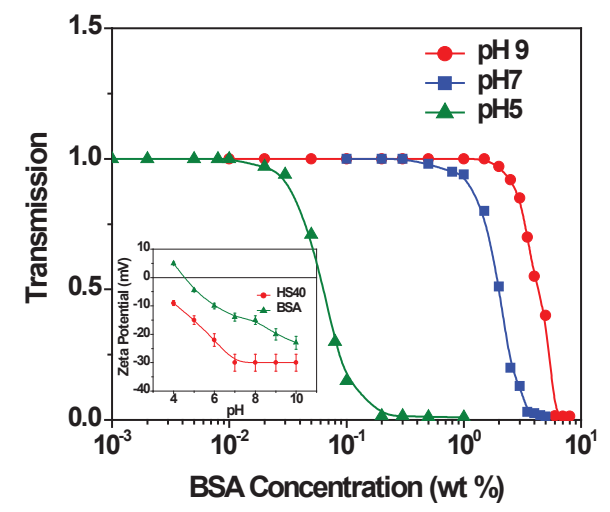

FIGURE 1. Phase behavior of 1 wt $\%$ HS40 silica nanoparticles with varying BSA concentration at different $\mathrm{pH}$. The inset shows the zeta potential as a function of $\mathrm{pH}$.

The structural evolution (kinetics) of the nanoparticles in presence of proteins was characterized by measuring the autocorrelation function and size distribution, using DLS. The intensity weighted size distributions of nanoparticle-BSA system at $\mathrm{pH} 9$ are shown in Figure 2. The distribution is bimodal in presence of BSA where the larger peaks represent the effective size of the nanoparticles and smaller peaks size of BSA protein. The mean value of nanoparticles and BSA is found to be $21 \mathrm{~nm}$ and $8 \mathrm{~nm}$, respectively. Interestingly the larger peak shifts towards the higher 
value with increasing the concentration of BSA. On the other hand, irrespective of the BSA concentration, the mean value of the smaller peak remains unchanged whereas the intensity of the smaller peak increases with BSA concentration. This presence of bimodal distribution also supports the fact that BSA does not adsorb on the silica nanoparticles. The structural evolution of the nanoparticles in presence of BSA finally leads to the aggregation of the nanoparticles above CPC, where the CPC monitored by DLS is found to be consistent with the phase behavior [Figure 1]. The nanoparticles, irrespective of the $\mathrm{pH}$, show similar features of evolution in respective phase behavior. However, the concentration of protein needed to get the required changes is lowered with decrease in $\mathrm{pH}$ because of the enhancement of depletion attraction. The interaction governing the phase behavior and the morphology of the aggregates have been characterized by small-angle neutron scattering (SANS).

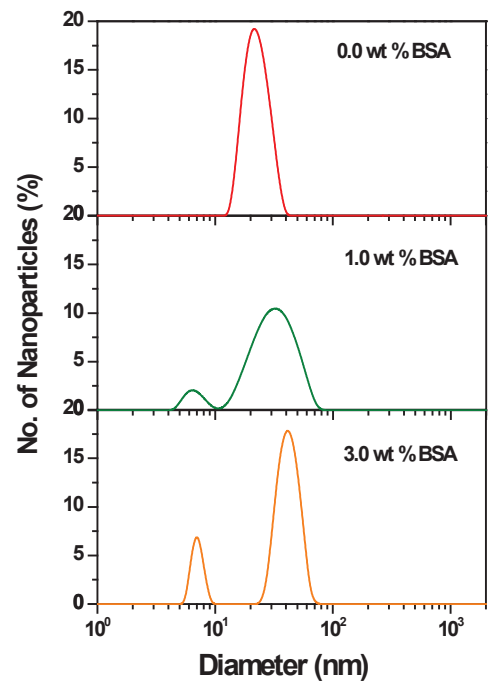

FIGURE 2. DLS study of structure evolution of $1 \mathrm{wt} \%$ HS40 silica nanoparticles in presence of BSA protein: particle size distribution with varying concentration of BSA protein at $\mathrm{pH} 9$.

Figure 3 shows the SANS data of $1 \mathrm{wt} \%$ silica nanoparticles in presence of 2 wt \% BSA protein at three different $\mathrm{pH}$. The data at higher $\mathrm{Q}$ is governed by the additive form factor of nanoparticles and protein and remain unaffected by $\mathrm{pH}$. The buildup in scattering intensity, in presence of BSA, at low Q is either due to evolution of attractive interaction and/or structure. The data for $\mathrm{pH} 9$ has been fitted with twoYukawa potential accounting the attractive as well as repulsive interaction [2]. The range of attractive interaction is found to be of the order of the BSA molecule. The linearity of the scattering profile, at low $Q$, for $\mathrm{pH} 7$ and $\mathrm{pH} 5$ indicates the fractal nature of the aggregates. The aggregates are found to be mass fractal having fractal dimension of about 2.3 and remain almost independent of the $\mathrm{pH}$. It should be noted that though the aggregates formed through diffusion limited cluster-cluster aggregation (DLCA) process have fractal dimension of about 1.8 in low density regime [4], the increase in fractal dimension with the density of the aggregating monomers has also been seen [5]. The observed fractal dimension 2.3 (greater than 1.8) is attributed to higher density of monomers for our experimental conditions. Thus the BSA induced aggregation of the nanoparticles is governed by DLCA process.

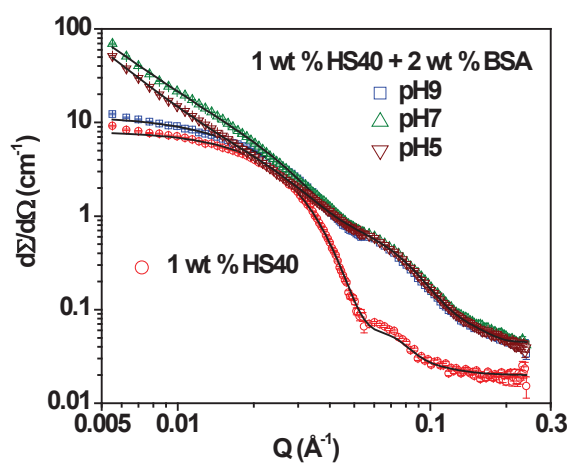

FIGURE 3. SANS data of $1 \mathrm{wt} \%$ HS40 Silica nanoparticles with $2 \mathrm{wt} \%$ BSA protein at different $\mathrm{pH}$.

\section{CONCLUSIONS}

The interaction of the globular model protein BSA with silica nanoparticle as a function of $\mathrm{pH}$ has been investigated by DLS and SANS. The dominance of electrostatic repulsion over site specific adsorption prevents the adsorption of BSA on the nanoparticles. The nonadsorption of BSA induces the depletion attraction between the nanoparticles whose strength increases with BSA concentration and finally leads to aggregation of the nanoparticles. The charge on BSA molecules can be tuned by the $\mathrm{pH}$ and hence the phase behavior. The formation of irreversible aggregates of nanoparticles at higher BSA concentration is governed by the diffusion-limited cluster-cluster aggregation process.

\section{REFERENCES}

1. I. Lynch and K. A. Dawson, Nano Today 3, 40 (2008).

2. I. Yadav, V. K. Aswal and J. Kohlbrecher, Phys. Rev. E 93, 052601 (2016).

3. S. Kumar, V. K. Aswal, and P. Callow, Langmuir 30, $1588(2014)$

4. M. Y. Lin, H. M. Lindsay, D. A. Weitz, R. C. Ball, R. Klein and P Meakin, Nature 339, 360 (1989).

5. M. D. Camejo, D. R. Espeso and L. L. Bonilla, Phys. Rev. E 90, 012306 (2014). 